\title{
Analysis of nocturnal actigraphic sleep measures in patients with COPD and their association with daytime physical activity
}

\author{
Gabriele Spina, ${ }^{1,2}$ Martijn A Spruit, ${ }^{3,4,5}$ Jennifer Alison, ${ }^{6,7}$ Roberto P Benzo, ${ }^{8}$ \\ Peter M A Calverley, ${ }^{9}$ Christian F Clarenbach, ${ }^{10}$ Richard W Costello, ${ }^{11}$ \\ David Donaire-Gonzalez, ${ }^{12,13,14}$ Selina Dürr, ${ }^{15}$ Judith Garcia-Aymerich, ${ }^{12,13,14}$ \\ Arnoldus J R van Gestel, ${ }^{10}$ Marco Gramm, ${ }^{16}$ Nidia A Hernandes, ${ }^{17}$ Kylie Hill, $^{18}$ \\ Nicholas S Hopkinson, ${ }^{19}$ Diana Jarreta, ${ }^{20}$ Malcolm Kohler, ${ }^{10}$ Anne M Kirsten, ${ }^{16}$ \\ Jörg D Leuppi, ${ }^{15}$ Helgo Magnussen, ${ }^{16}$ François Maltais, ${ }^{21}$ William D-C Man, ${ }^{19}$ \\ Zoe J McKeough, ${ }^{6}$ Rafael Mesquita, ${ }^{3,22}$ David Miedinger, ${ }^{15}$ Fabio Pitta, ${ }^{17}$ \\ Sally J Singh, ${ }^{23}$ Frank W J M Smeenk, ${ }^{24}$ Ruth Tal-Singer, ${ }^{25}$ Barbara Vagaggini, $^{26}$ \\ Benjamin Waschki, ${ }^{16}$ Henrik Watz, ${ }^{16}$ Emiel F M Wouters, ${ }^{3,22}$ Stefanie Zogg, $^{15}$ \\ Albertus C den Brinker ${ }^{2}$
}

\begin{abstract}
- Additional material is published online only. To view please visit the journal online (http://dx.doi.org/10.1136/ thoraxjnl-2016-208900)
\end{abstract}

For numbered affiliations see end of article.

Correspondence to Gabriele Spina, Dr Data Science Group, Philips Research, High Tech Campus 34, Eindhoven 5656 AE, The Netherlands; gabriele.spina@philips.com

Received 12 May 2016 Revised 15 November 2016 Accepted 7 December 2016 Published Online First 12 January 2017

\section{SLinked}

- http://dx.doi.org/10.1136/ thoraxjnl-2016-209905

CrossMark

To cite: Spina G, Spruit MA, Alison J, et al. Thorax 2017;72:694-701

\section{ABSTRACT}

Background Sleep disturbances are common in patients with chronic obstructive pulmonary disease (COPD) with a considerable negative impact on their quality of life. However, factors associated with measures of sleep in daily life have not been investigated before nor has the association between sleep and the ability to engage in physical activity on a day-to-day basis been studied.

Aims To provide insight into the relationship between actigraphic sleep measures and disease severity, exertional dyspnoea, gender and parts of the week; and to investigate the association between sleep measures and next day physical activity.

Methods Data were analysed from 932 patients with COPD ( $66 \%$ male, $66.4 \pm 8.3$ years, $\mathrm{FEV}_{1} \%$ predicted $=50.8 \pm 20.5$ ). Participants had sleep and physical activity continuously monitored using a multisensor activity monitor for a median of 6 days. Linear mixed effects models were applied to investigate the factors associated with sleep impairment and the association between nocturnal sleep and patients' subsequent daytime physical activity.

Results Actigraphic estimates of sleep impairment were greater in patients with worse airflow limitation and worse exertional dyspnoea. Patients with better sleep measures (ie, non-fragmented sleep, sleeping bouts $\geq 225 \mathrm{~min}$, sleep efficiency $\geq 91 \%$ and time spent awake after sleep onset $<57 \mathrm{~min}$ ) spent significantly more time in light $(p<0.01)$ and moderate-to-vigorous physical activity $(p<0.01)$.

Conclusions There is a relationship between measures of sleep in patients with COPD and the amount of activity they undertake during the waking day. Identifying groups with specific sleep characteristics may be useful information when designing physical activityenhancing interventions.

\section{Key messages}

What is the key question?

- Are actigraphic sleep measures associated with disease severity, exertional dyspnoea, gender and parts of the week (ie, weekdays vs weekends); and is there an association between assessed sleep measures and next day physical activity?

What is the bottom line?

- Actigraphic sleep measures in patients with COPD were found (1) to be worse in patients with severe airway obstruction and dyspnoea and (2) to be associated with the amount of physical activity the subjects undertake during the following waking day.

Why read on?

- With poor sleep and physical inactivity each recognised as key public health priorities, this research has shown that poor sleep, assessed under free living conditions, may hamper efforts of patients with COPD to be physically active, emphasising a relationship between sleep and daily physical activity.

\section{INTRODUCTION}

In addition to progressive chronic airflow limitation, patients with chronic obstructive pulmonary disease (COPD) commonly have multiple extrapulmonary effects and comorbidities, which are associated with physical inactivity. ${ }^{1}$ Although there is general agreement about the need to assess and improve physical activity in people with COPD, the factors associated with patients' capability to 
engage in physical activity are not well established, which may limit the impact of physical activity enhancement interventions. ${ }^{2}$

Sleep disturbance, such as sleep fragmentation during the night, is common in patients with COPD ${ }^{3}$ and is a major complaint after dyspnoea and fatigue. ${ }^{4}$ Despite the high prevalence of disturbed sleep in COPD, night-time symptoms are often underestimated and are not a focus of current disease management. ${ }^{3}$

Activity-based or actigraphy sleep-wake monitoring is a costeffective method to assess sleep that has gained a central role in clinical sleep research and sleep medicine $e^{5}$ by proving invaluable insights for the improvements of individualised treatment decisions. ${ }^{6}$ Although actigraphy should not be viewed as a substitute for sleep diaries or overnight polysomnography, it can provide useful information about sleep in the natural sleep environment if used for extended period of time with minimal participant burden. $^{7}$

Nocturnal sleep, studied by actigraphy, has been shown to be markedly impaired in patients with COPD compared with controls. ${ }^{8}$ However, there is scant and discordant information on whether actigraphic measures of sleep worsen as the severity of dyspnoea and airflow limitation increases. ${ }^{8}{ }^{9}$ Therefore, more data are needed for a better understanding of the factors associated with sleep impairment in patients with COPD. Moreover, although sleep disturbance likely contributes to daytime symptoms like chronic fatigue, lethargy and overall impairment in quality of life described by these patients, ${ }^{10}$ no published study has objectively investigated the association between night-time sleep measures and measures of spontaneous physical activity the following day.

In this study, data were pooled from different studies resulting in a large sample of patients with mild to very severe COPD who had extended objective measures of sleep and physical activity during daily life assessed using a multisensor activity monitor. These data were used to (1) provide insight into the relationship between objectively determined sleep measures and disease severity, exertional dyspnoea, gender and parts of the week (ie, weekdays vs weekends); and (2) investigate whether there was an association between objectively assessed sleep measures and next day physical activity. Our hypotheses were that patients with more severe COPD defined according to the Global Initiative for Chronic Obstructive Lung Disease $(G O L D)^{11}$ criteria and higher Modified Medical Research Council (MMRC) ${ }^{12}$ dyspnoea score would have more objectively measured sleep disturbances, and that nights of impaired sleep would be followed by days characterised by lower levels of physical activity.

\section{METHODS}

\section{Study design and participants}

In this retrospective, cross-sectional study, data from previous studies (details can be found in the online supplementary appendix pp. 3-5) were obtained from research groups in 10 countries: Europe (the Netherlands, the UK, Switzerland, Germany, Italy, Ireland, Spain), North America (the USA), South America (Brazil) and Oceania (Australia). Criteria to include participants in the current analyses were clinically stable COPD (ie, stable shortness of breath and sputum production) with a postbronchodilator ratio of $\mathrm{FEV}_{1}$ to $\mathrm{FVC}<0.70$; no COPD exacerbations within the last 30 days; and availability of sleep and daytime physical activity baseline data recorded in daily life using the SenseWear Armband (SWA) or SenseWear Mini Armband activity monitors (BodyMedia, Pittsburgh, Pennsylvania, USA) before any specific interventions were undertaken. The SWA has been previously used to study the circadian pattern of sleep and skin temperature ${ }^{13}$ to assess sleep pattern in patients with acute coronary disease ${ }^{14}$ and to monitor sleep in COPD. ${ }^{15-17}$ Data from 1384 patients were available for the present study.

\section{Data recordings}

Study participants wore the SWA on the upper arm both during daytime and night-time so that continuous data related to sleep and real-life activities were recorded.

While worn, the SWA recorded continuously longitudinal and transverse acceleration, skin temperature, near body temperature, heat flux and galvanic skin response. Collected data sent to a PC were used to calculate the metabolic equivalent of task (MET), number of steps, posture (lying down vs not lying down) and sleeping status (sleep vs wakefulness) on a minute-by-minute basis. ${ }^{18}$ The use of multisensory data in combination with pattern recognition algorithms ensured that the MET estimation was insensitive to noise and random motion artefacts $^{19}$ and allowed the objective assessment of sleep ${ }^{20}$ and daytime activity levels. ${ }^{21}$

METs data were divided into activity intensity levels using the thresholds proposed by the American College of Sports Medicine: very light intensity, <2.0 METs; light intensity, 2.02.9 METs; and moderate-to-vigorous intensity, $\geq 3.0$ METs. ${ }^{22}$

Time in bed and time out of bed were derived from the minutes coded by the activity monitor as 'sleeping' and 'lying down' using a custom-made algorithm (online supplementary appendix pp. 6-9). Based on these data, the following nighttime and daytime sleep measures were derived: total night sleeping time, number of nocturnal sleeping bouts, duration of nocturnal sleeping bouts, sleep efficiency, wake after sleep onset, total day sleeping time, number of daytime sleeping bouts and average duration of daytime sleeping bouts. Sleeping bouts were defined as consecutive minutes marked by the sensor as sleeping. In this study, 'sleep quality' is used to refer to the collection of these sleep measures with definitions presented in table 1.

As physical activity measures, the number of steps performed during daytime and the time spent in very light, light and moderate-to-vigorous activities was computed for each assessed day.

Participants who wore the SWA for at least 22 hours per day, with a minimum of four assessed consecutive days (two weekdays + Saturday+Sunday) were included. ${ }^{21}$ Participants who did not have their sleep regularly distributed during night-time or who had $<4$ hours of time in bed were excluded in order to minimise possible errors due to methodological limitations. For these patients, it was not possible to find a reliable estimation of the time in bed and time out of bed as explained in the online supplementary appendix pp. 6-9.

\section{Statistical analysis}

Since physical activity can vary from day to day, complex effects and interactions are expected to occur, which creates the need for multiple days of measurements and proper statistical approaches to identify associations with sleep. Linear mixed-effect models were used to study (1) which factors influenced sleep quality measures and (2) whether and to what extent sleep quality measures were associated with subsequent daily physical activity levels and daytime sleep. This kind of multilevel analysis was chosen to account for repeated measurements and control for several confounding factors such as disease and dyspnoea severity, current smoking status at the time of enrollment, country, gender, parts of the week (ie, 
Table 1 Nocturnal and daytime sleep measures derived from actigraphy data

\begin{tabular}{|c|c|c|}
\hline Variable name & Abbreviation & Description \\
\hline Total night sleeping time & TNST & Total night sleeping time is calculated as the sum of all minutes scored as sleep during time in bed. \\
\hline Number of nocturnal sleeping bouts & NNSB & Number of nocturnal sleeping bouts during time in bed. A higher NNSB indicates more fragmented sleep. \\
\hline Duration of nocturnal sleeping bouts & DNSB & $\begin{array}{l}\text { Average duration of nocturnal sleeping bouts during time in bed. A higher DNSB indicates longer sleeping bouts } \\
\text { and, in turn, less nocturnal sleeping disturbances. }\end{array}$ \\
\hline Sleep efficiency & Seff & Sleep efficiency defined as the ratio of TNST and time in bed. \\
\hline Wake after sleep onset & WASO & Time spent awake during time in bed after the first nocturnal sleep onset. \\
\hline Total day sleeping time & TDST & Total day sleeping time defined as the total time spent asleep during the out of bed period. \\
\hline Number of daytime sleeping bouts & NDSB & Number of daytime sleeping bouts indicates how many naps a patient takes during the day. \\
\hline Duration of daytime sleeping bouts & DDSB & Average duration of daytime sleeping bouts during the day. A higher DDSB indicates longer naps. \\
\hline
\end{tabular}

weekdays and weekends), age and body mass index (BMI). To construct the models, standard statistical packages were used (details can be found in the online supplementary appendix $\mathrm{p}$ 12). Similar analyses were carried out in refs. 23-25. Least-squares means (LS-means) and differences of LS-means of the fixed effects were calculated to present the results. Degrees of freedom and $\mathrm{p}$ values for significant differences (significant if $\mathrm{p}<0.05$ ) were computed using Satterthwaite's approximation. ${ }^{26}$ Comparisons of demographic and clinical characteristics between included and excluded patients were evaluated by Mann-Whitney $U$ test for continuous variables and $\chi^{2}$ test for categorical variables. Analyses were carried out using MATLAB R2015a (The MathWorks, Natick, Massachusetts, USA) and R (R Core Team, 2012) software.

\section{RESULTS}

In total, 932 patients with COPD were eligible for analysis. Figure 1 shows the flow of participants through the study. Patients excluded due to irregular sleeping patterns and not enough time in bed had significantly lower $\mathrm{FEV}_{1} \%$ predicted compared with included patients $(46.6 \pm 19.4$ vs $50.8 \pm 20.5$, $\mathrm{p}<0.05)$. No significant differences between included and excluded patients were observed for age, gender, BMI, smoking status at enrollment and MMRC. The median number of days analysed per patient was six (four weekdays+Saturday and Sunday), resulting in a total of 5646 valid assessed days, of which $3788(67 \%)$ were weekdays. Demographic and clinical characteristics of the patients included in the study are presented in table 2 (those of the excluded patients can be found in online supplementary materials table $\mathrm{S} 1$, appendix $\mathrm{p} 11$ ).

\section{Sleep measures evaluation in patients with COPD}

Figure 2 shows the associations of night-time and daytime sleep measures with disease severity, dyspnoea, gender and parts of the week. In particular, the total night sleeping time (figure $2 \mathrm{~A}$ ) was significantly higher in women $(p<0.01)$ and during weekends $(p<0.01)$. The number of nocturnal sleeping bouts (figure $2 \mathrm{~B}$ ) increased both with airflow limitation and exertional dyspnoea. Patients with the most severe airflow limitation and exertional dyspnoea had significantly more fragmented sleep than patients with the lowest GOLD grade $(p<0.01)$ and MMRC score $(p<0.05)$. The number of nocturnal sleeping bouts was significantly higher in men $(p<0.001)$ and during weekends $(p<0.05)$. Both short duration of nocturnal sleeping bouts and low sleep efficiency were associated with a higher GOLD grade and dyspnoea score (figure 2C, D). Patients with the most severe airflow limitation had significantly shorter sleeping bouts $(p<0.05)$ and lower sleep efficiency $(p<0.01)$ than

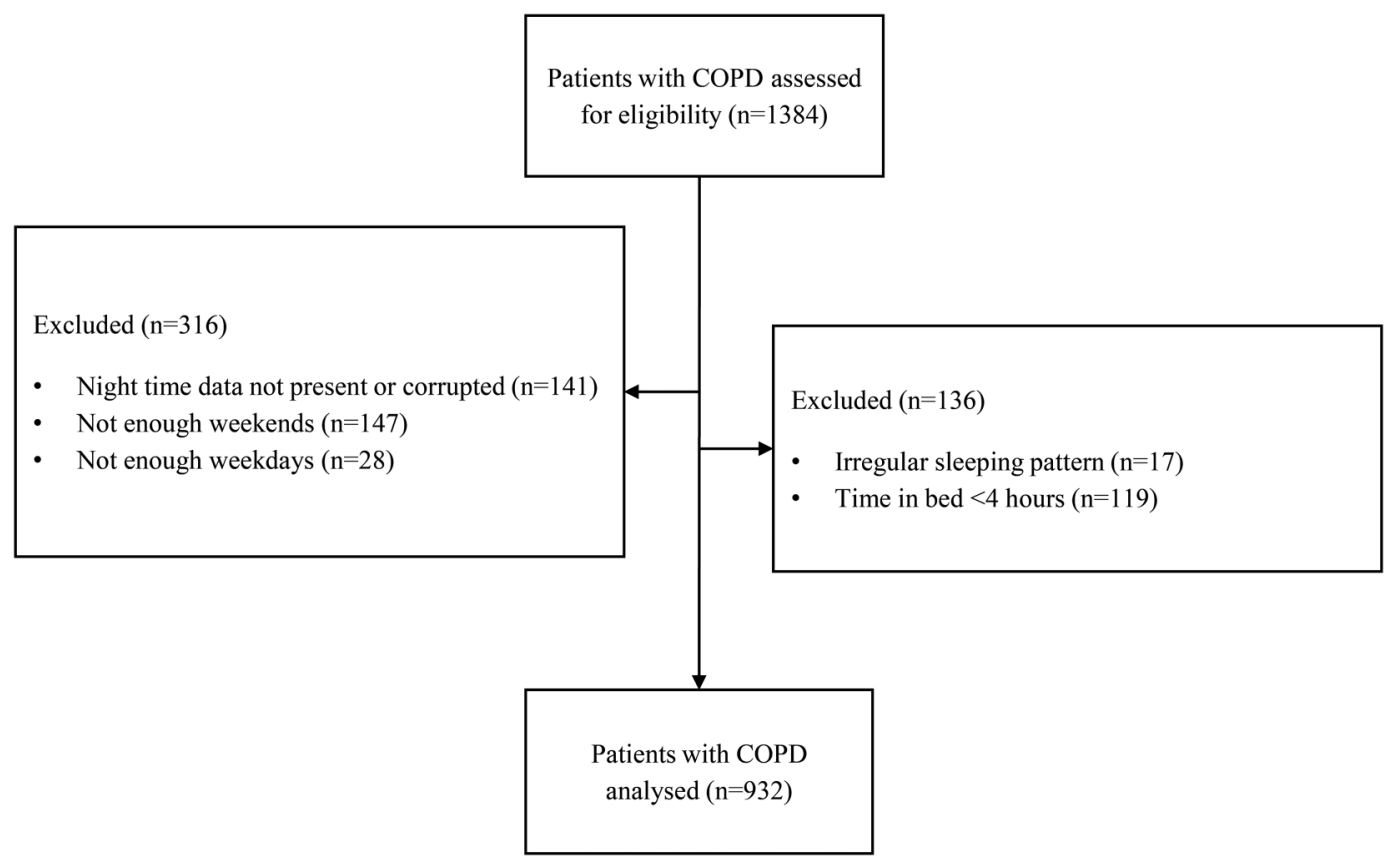

Figure 1 Flow of patients through the study. 
Table 2 Demographic and clinical characteristics

Included patients $(n=932)$
Male/female (\%)

Smokers ${ }^{*} /$ never or former smokers ${ }^{\dagger}(\%)$

Age (year)

BMI $\left(\mathrm{kg} / \mathrm{m}^{2}\right)$

FEV $1 \%$ predicted

GOLD 1-2-3-4 (\%)

MMRC $^{\ddagger} 0-1-2-3-4(\%)$
$66 / 34$

$33 / 67$

$66.4 \pm 8.3$

$26.3 \pm 5.4$

$50.8 \pm 20.5$

$10-40-32-18$

$14-27-22-18-6$
Data in the table are expressed as percentages, or means \pm SD.

${ }^{*}$ Current smokers at the time of enrollment.

${ }^{\dagger}$ Never or former smokers at the time of enrollment.

¥MMRC data for 805 patients.

BMI, body mass index; GOLD, Global Initiative for Chronic Obstructive Lung Disease;

MMRC, Modified Medical Research Council.

patients classified as GOLD 3. Patients reporting the highest MMRC score had significantly shorter sleeping bouts $(p<0.05)$ and lower sleep efficiency $(\mathrm{p}<0.01)$ compared with patients with lower MMRC scores. Duration of sleeping bouts and sleep efficiency was significantly higher for women $(p<0.001)$. The time spent awake after the first sleep onset (figure 2E) increased both with disease severity and dyspnoea, being worst in patients with GOLD grade $4(\mathrm{p}<0.05)$ and MMRC score $4(\mathrm{p}<0.01)$.

During daytime (figure $2 \mathrm{~F}-\mathrm{H}$ ), the sleeping time, the number and duration of sleeping bouts increased significantly with dyspnoea $(p<0.05)$. Sleeping time and duration of sleeping bouts were significantly shorter in patients with GOLD grade 4 compared with patients classified as GOLD $3(\mathrm{p}<0.05)$.

\section{Association between objective sleep measures and daytime physical activity}

Nocturnal sleep measures were divided into quartiles $\left(Q_{1}\right.$, shortest/lowest; $\mathrm{Q}_{4}$, longest/highest) to assess the association with daytime physical activity measures (see online supplementary materials table S2, appendix p 13).

As shown in figure $3 \mathrm{~A}$, the number of steps performed during the day was inversely related to the number of sleeping bouts and the minutes spent awake after the sleep onset. On the other hand, the number of steps increased as the duration of the sleeping bouts and the sleep efficiency increased. In particular, patients who had their sleep characterised by one sleeping bout, long sleeping bouts $(\geq 225 \mathrm{~min})$, high sleep efficiency $(\geq 91 \%)$ and low time spent awake after the sleep onset $(<57 \mathrm{~min})$ performed, on average, 600 steps more on the following day with respect to patients who had more fragmented sleep ( $\geq 4$ bouts), lower sleep efficiency $(<71 \%)$, lower sleeping bout durations $(<86 \mathrm{~min})$ and higher time spent awake after the sleep onset $(\geq 165 \mathrm{~min}) \quad(p<0.001$ for all $)$. Patients who slept $>480 \mathrm{~min}$ performed a smaller number of steps than the other patients, but no significant differences were found if the number of steps of each patient is divided by the time spent awake (data not shown). As presented in figure $3 \mathrm{~B}$, the percentage of daytime spent in very light activities was higher in patients who slept $>480 \mathrm{~min}$ and it increased with sleep fragmentation, and time spent awake after the sleep onset. Furthermore, the time spent in very light activities decreased as sleep efficiency and duration of sleeping bouts increased. Patients with the worst nocturnal sleep measures (ie, sleep characterised by sleeping bouts $<86 \mathrm{~min}$, sleep efficiency $<71 \%$ and time spent awake after the sleep onset $\geq 165 \mathrm{~min}$ ) spent on average the $74 \%$ of the time awake in very light-intensity activities, $1 \%$ more than patients with sleep characterised by sleeping bouts $\geq 225 \min (\mathrm{p}<0.01)$, sleep efficiency $\geq 91 \%(p<0.001)$ and time spent awake after the sleep onset $<57 \min (\mathrm{p}<0.01)$.

Patients with the smallest number of sleeping bouts per night, longest sleeping bouts, highest sleep efficiency and shortest time spent awake during the night spent more time in light (figure 3C) and moderate-to-vigorous (figure 3D) physical activities on the following day $(\mathrm{p}<0.01$ for all). In particular, compared with patients with the worst sleep measures, these patients spent on average $9 \mathrm{~min}$ more in light-intensity activities and $8 \mathrm{~min}$ more in moderate-to-vigorous activities that correspond to the $26 \%$ of the recommended daily amount. ${ }^{27}$

Daytime sleep was inversely related to the amount of nocturnal sleep, the duration of sleeping bouts and the sleep efficiency (figure 4). Patients who showed relatively short sleep duration during the night $(<347 \mathrm{~min})$, who had short sleeping bouts (<86 min) and low sleep efficiency $(<71 \%)$ reported more daytime sleep compared with subjects who slept $>480 \mathrm{~min}$ during the night $(\mathrm{p}<0.001)$, had sleeping bouts $>225 \mathrm{~min}$ $(\mathrm{p}<0.05)$ and a sleep efficiency $>91 \%(\mathrm{p}<0.05)$.

\section{DISCUSSION}

We describe, to our knowledge, the first study in a large sample of participants with COPD, targeting the association of objectively measured sleep impairment with airflow limitation, exertional dyspnoea, gender, parts of the week and daytime activity levels.

We found that objective sleep quality measures are worse in patients with severe airway obstruction and dyspnoea. This is in agreement with George and Bayliff, ${ }^{28}$ who showed that subjective sleep disturbances tend to be more severe with advanced COPD, and with Dodge et al, ${ }^{29}$ who showed that patients with dyspnoea are more likely to report persistent sleep disturbance. Conversely, Hartman et $a l^{9}$ failed to find a significant association between night's rest parameters assessed by an accelerometer and both airflow limitation and dyspnoea. However, this latter study did not evaluate sleep quality measures, but rather quantitative parameters such as body movements and number of posture transitions that are not necessarily related to poor sleep quality. ${ }^{9}$ Nunes et $a l^{8}$ reported that the MMRC score was an important predictor of objectively measured sleep quality in 26 patients with moderate to very severe COPD. However, the small sample size precluded any firm conclusions on the differences between patients with different dyspnoea scores.

The strong evidence provided by our findings regarding the association of sleep impairment with the most severe stage of the disease and dyspnoea could be explained by several potential mechanisms. First, it is intuitively apparent that because patients with severe COPD have a lower oxygen saturation during wakefulness, the normal ventilation changes that occur with sleep exacerbate this pre-existing state to the point of severity impairing sleep. ${ }^{30}$ Second, the lying sleeping position assumed by the participants in the study and the reduction in muscle tone causes increased upper airway resistance. ${ }^{10} 30$ This might have more impact in patients who are least able to tolerate any further challenge to their ventilatory capability. Moreover, patients with severe COPD frequently experience episodes of worsening symptoms that may also preclude from adequate sleep.

Participants with the most severe disease had worse sleep but they did not seem to compensate it during the day by adding naps. Most dyspnoeic patients, in turn, seemed to do so. Other investigators have also found that daytime sleepiness was related more strongly to the presence of respiratory symptoms than to a diagnosis of lung disease or the degree of airway obstruction. ${ }^{31}$

We saw significant gender differences after correction for age, BMI, smoking status at enrollment, country of origin, 

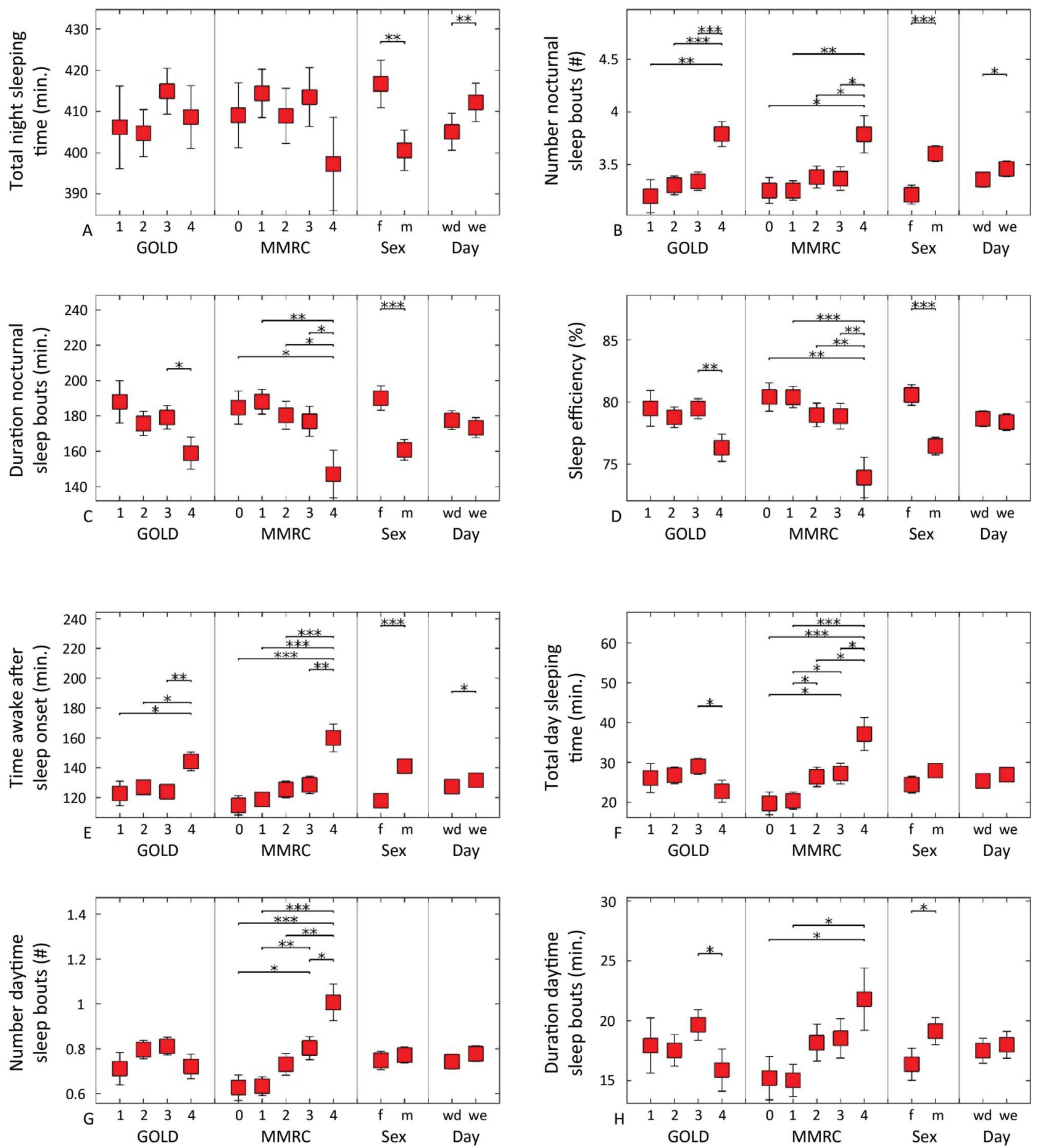

Figure 2 Impact of disease severity according to the Global Initiative for Chronic Obstructive Lung Disease (GOLD) grades, daytime dyspnoea according to the Modified Medical Research Council (MMRC) scale, sex ( $f=f e m a l e, ~ m=m a l e)$, and parts of the week (wd=weekdays, we=weekends) on sleep parameters ((A) total night sleeping time; (B) number of nocturnal sleeping bouts; $(C)$ average duration of nocturnal sleeping bouts; (D) sleep efficiency; (E) time awake after the first sleep onset; (F) total day sleeping time; $(G)$ number of daytime sleeping bouts; $(\mathrm{H})$ average duration of daytime sleeping bouts). Data are expressed as least-square means \pm SE. Significance levels for pairwise comparisons are indicated as horizontal bars with ${ }^{*} p<0.05,{ }^{* *} p<0.01$ and ${ }^{* * *} p<0.001$.

GOLD and MMRC. Men had worse sleep quality measures and spent more time sleeping during the day than women. There is currently only limited information regarding the influence of gender on sleep in COPD. In a previous study using actigraphy, no significant gender-related differences in sleep quality were found. ${ }^{8}$ Other studies have documented that women appear to report more sleep-related complaints. ${ }^{32}$ However, objective and subjective assessments of sleep seem to reflect different aspects and need therefore not be necessarily in agreement. $^{33}$

During weekends, patients had significant more fragmented sleep and spent significantly more time awake after the first sleep onset than during weekdays. Our results showed that impaired sleep during weekends was compensated by sleeping longer.

This study showed that nights of lower sleep quality were followed by days of lower levels of physical activity in people with 

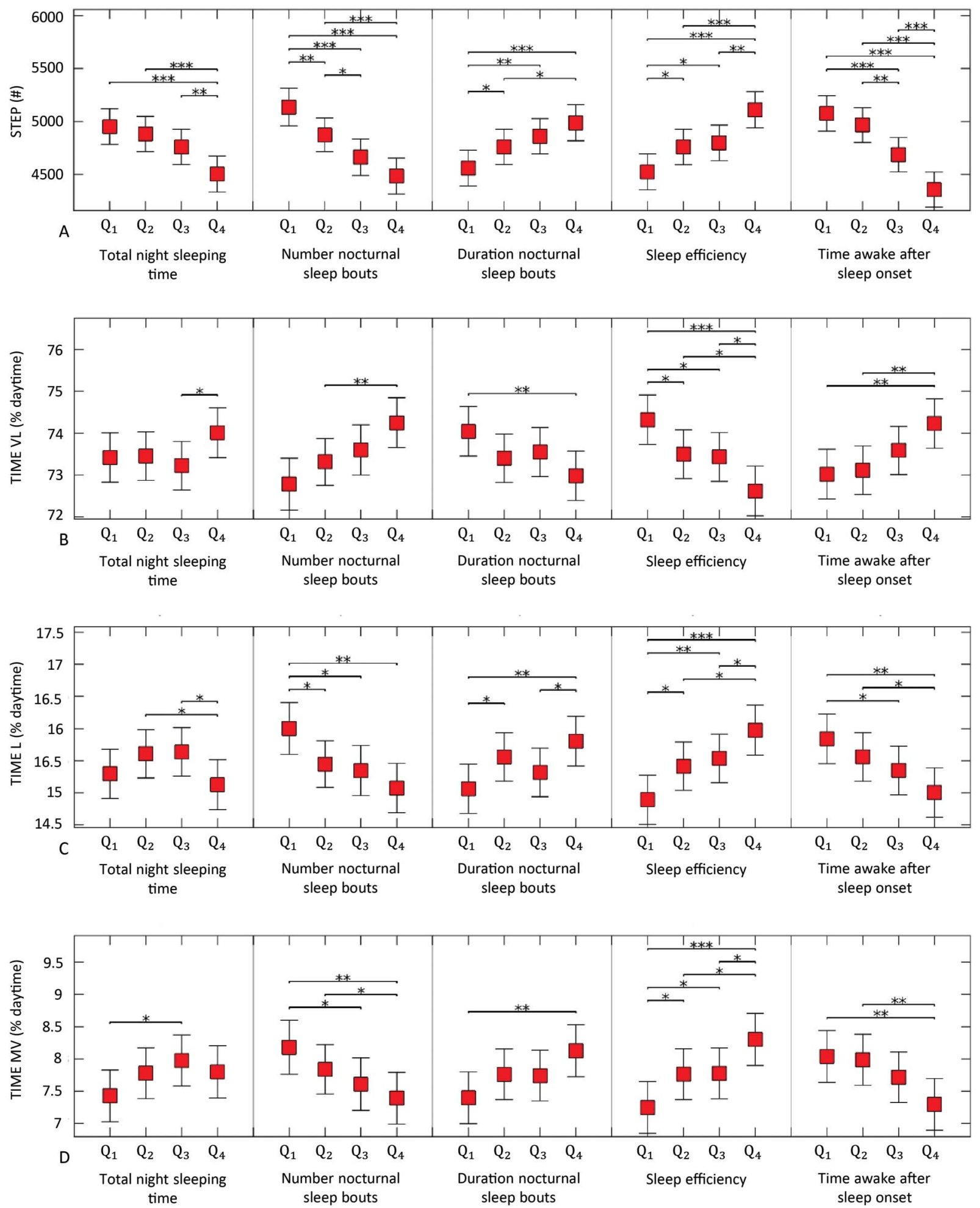

Figure 3 Association between nocturnal sleep parameters (total night sleeping time; number of nocturnal sleeping bouts; average duration of nocturnal sleep bouts; sleep efficiency; time awake after the first sleep onset) and daytime physical activity measures ((A) STEP=steps performed; (B) TIME VL=time spent in very light activity; (C) TIME L=time spent in light activity; (D) TIME MV=time spent in moderate-to-vigorous activity). Data are expressed as least-square means $\pm \mathrm{SE}$. Significance levels for pairwise comparisons are indicated as horizontal bars with ${ }^{*} p<0.05$, ${ }^{* *} p<0.01$ and ${ }^{* * *} \mathrm{p}<0.001$. TIME VL, TIME L and TIME MV are presented in $\%$ of the out of bed time.

COPD. It is important to note that the potential influence of airflow limitation, severity of dyspnoea, smoking status at enrollment, country, gender, parts of the week (ie, weekdays and weekends), age and BMI was taken into account since these variables were included in the multilevel models used.
The association of impaired sleep with worse daytime performance is consistent with findings showing that it plays a substantial role in reduced quality of life in patients with COPD. ${ }^{34} 35$ Moreover, these findings are consistent with other correlational evidence drawn from older adults, suggesting that objectively 


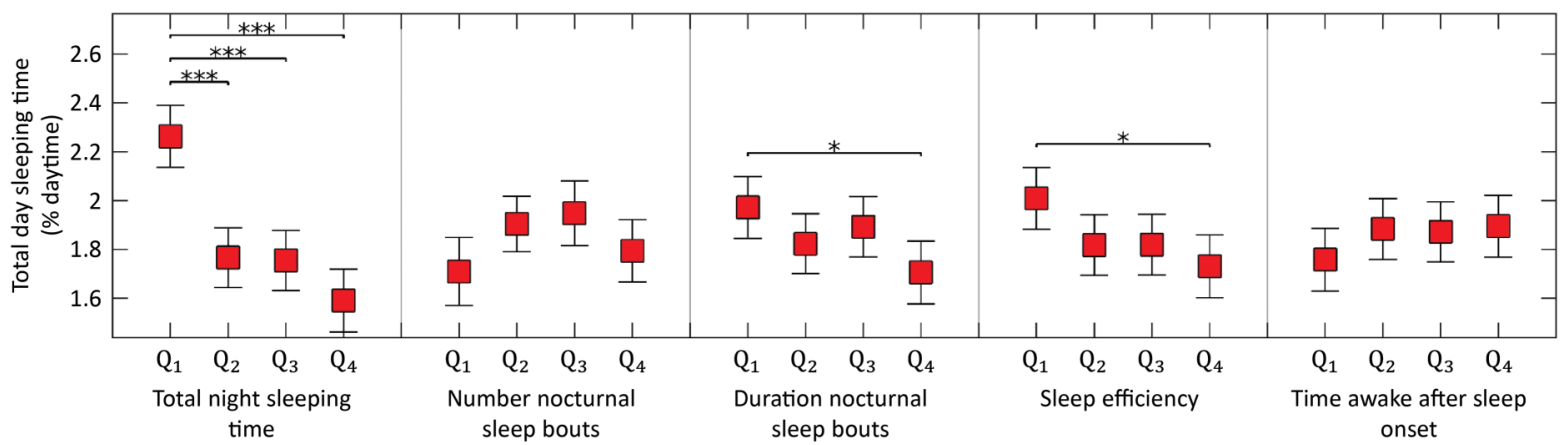

Figure 4 Association between nocturnal sleep parameters (total night sleeping time; number of nocturnal sleeping bouts; average duration of nocturnal sleeping bouts; sleep efficiency; time awake after the first sleep onset) and total day sleeping time. Data are expressed as least-square means \pm SE. Significance levels for pairwise comparisons are indicated as horizontal bars with ${ }^{*} p<0.05,{ }^{* *} p<0.01$ and ${ }^{* * *} p<0.001$. Total day sleeping time is presented in \% of the out of bed time.

measured poor sleep was associated with worse daytime physical function evaluated in clinical settings, ${ }^{36} 37$ yet none of these studies demonstrates a direct effect of sleep on subsequent physical activity during daily life. Although inferring a causal relationship was out of the scope of this study, our finding provided a good illustration of the sequential association as it incorporated a clear chronological order of the predictor (sleep quality measure) and the predicted variable (physical activity). However, the possibility that poor daytime performance may be a causative factor for poor sleep quality should be considered. In this regard, it has been previously shown that patients who are most hypoxaemic when awake are most severely hypoxaemic when asleep with the consequence of having both reduced physical activity during the day and sleep disturbance during the night. On the contrary, in patients with mild (or absent) daytime hypoxaemia, this nocturnal desaturation is, in most cases, slight to moderate. ${ }^{30}$

The deficit in good sleep, in association with changes of sleep architecture, may make sleep not as restorative as needed and may cause a significant sleep deficit. ${ }^{38}$ Accordingly, our study provides evidence to suggest that poor sleep efficiency and short sleeping bouts during the night are possibly compensated by additional daytime naps.

The fact that poor sleep quality was associated with reduced physical activity levels may have important consequences with regard to current clinical practice. First, our findings suggest the possibility of increasing spontaneous engagement in physical activity through improving sleep since, in the absence of any intervention, patients having had a better night of sleep spontaneously engaged in more physical activity the following day. Second, existing strategies for promoting physical activity tend to focus on actions during the day. Additional efforts in promoting quality sleep among physically inactive subgroups may increase the overall impact of these interventions. Finally, sleep should be assessed and taken into account when analysing physical activity in COPD, although recent literature is often overlooking the continuity between night-time sleep and daytime physical activity. ${ }^{39}$

This study has some limitations that should be considered when interpreting the results. First, data regarding exacerbations, hospitalisations frequency and smoking history prior to enrolment were not available, which prevented to investigate their potential impact on daytime physical activity and sleep disruption. Our participants were not screened for sleep-related disorders, such as obstructive sleep apnoea (OSA). However, because COPD and OSA are both common chronic conditions, these should be expected to occur together, particularly among overweight individuals with COPD. ${ }^{40}$ Moreover, no significant differences were previously found in measures of dyspnoea, sleep quality, sleep efficiency and sleepiness during the day between patients with COPD only and patients with COPD-OSA overlap. ${ }^{40}$ Although we cannot know whether the patients included had OSA, we can rule out that specific interventions influenced quality or quantity of sleep since only baseline data were analysed.

To the current authors' knowledge, the SWA has not been properly validated to study sleep in COPD. However, several days of assessment reduce the inherent measurement errors in actigraphy and further increase the reliability. ${ }^{5}$ Moreover, although it has been shown to be a reliable method for determining sleep in patients with sleep-breathing disorders when compared against polysomnography, ${ }^{20}$ the SWA might underestimate the time spent sleeping for those patients who sleep in a sitting position in order to facilitate their breathing.

In summary, sleep impairment in patients with COPD tends to be more pronounced in patients with severe airflow limitation and in those with worse exertional dyspnoea. Moreover, nocturnal sleep impairment appears to be an important factor associated with the capability to engage in physical activity on a day-to-day basis. In particular, nights of better sleep quality measures were followed by days of higher levels of physical activity. Further research is needed to identify the causal association between night-time sleep and the decline in daytime physical activity, as well as to assess whether the management of nighttime symptoms and the reduction of sleep impairment can facilitate the adoption of a physically more active lifestyle in patients with COPD. Considering both our current understanding of the negative health consequences of sleep disturbance in COPD and the current limited efficacy of interventions in significantly improving and maintain physical activity enhancement, our data suggest that approaches to improve sleep need to be considered as additional targets for tailored interventions in patients with COPD since it may have significant implications for facilitating greater adherence and a favourable impact on lifestyle.

\section{Author affiliations}

${ }^{1}$ Department of Signal Processing Systems, Technische Universiteit Eindhoven, Eindhoven, The Netherlands

${ }^{2}$ Data Science Group, Philips Research, Eindhoven, The Netherlands

${ }^{3}$ Department of Research and Education, CIRO+, Center of Expertise for Chronic Organ Failure, Horn, The Netherlands

${ }^{4}$ REVAL - Rehabilitation Research Center, BIOMED - Biomedical Research Institute, Faculty of Medicine and Life Sciences, Hasselt University, Diepenbeek, Belgium ${ }^{5}$ Department of Respiratory Medicine, Maastricht University Medical Centre, NUTRIM School of Nutrition and Translational Research in Metabolism, Maastricht, The Netherlands

${ }^{6}$ Clinical and Rehabilitation Sciences, The University of Sydney, Sydney, New South Wales, Australia

${ }^{7}$ Physiotherapy Department, Royal Prince Alfred Hospital, Sydney, New South Wales, Australia 
${ }^{8}$ Mindful Breathing Laboratory, Mayo Clinic, Rochester, Minnesota, USA

${ }^{9}$ School of Ageing and Chronic Disease, University Hospital Aintree, Liverpool, UK

${ }^{10}$ Pulmonary Division, University Hospital of Zurich, Zurich, Switzerland

${ }^{11}$ Department of Respiratory Medicine, Beaumont Hospital, Dublin, Ireland

${ }^{12}$ Centre for Research in Environmental Epidemiology (CREAL), Barcelona, Spain

${ }^{13}$ CIBER Epidemiología y Salud Pública (CIBERESP), Barcelona, Spain

${ }^{14}$ Universitat Pompeu Fabra (UPF), Barcelona, Spain

${ }^{15}$ Medical University Clinic, Cantonal Hospital Baselland, Liestal and Medical Faculty, University of Basel, Basel, Switzerland

${ }^{16}$ Pulmonary Research Institute at Lung Clinic Grosshansdorf, Airway Research

Center North, Member of the German Centre for Lung Research, Grosshansdorf, Germany

${ }^{17}$ Laboratory of Research in Respiratory Physiotherapy, Department of Physiotherapy, State University of Londrina (UEL), Londrina, Brazil

${ }^{18}$ School of Physiotherapy and Exercise Science, Curtin University, Perth, Washington, Australia

${ }^{19}$ NIHR Respiratory Biomedical Research Unit, Royal Brompton \& Harefield NHS

Foundation Trust and Imperial College, London, UK

${ }^{20}$ AstraZeneca, Barcelona, Spain

${ }^{21}$ Centre de recherche, Institut Universitaire de cardiologie et de pneumologie de

Québec, 2725 Chemin Ste-Foy Québec, Université Laval, Québec, Canada

${ }^{22}$ Department of Respiratory Medicine, Maastricht University Medical Center+

(MUMC+), Maastricht, The Netherlands

${ }^{23}$ Centre for Exercise and Rehabilitation Science, University Hospitals of Leicester

NHS Trust, Leicester, UK

${ }^{24}$ Department of Respiratory Medicine, Catharina Hospital, Eindhoven, The

Netherlands

${ }^{25}$ GSK R\&D, King of Prussia, Pennsylvania, USA

${ }^{26}$ Cardio-Thoracic and Vascular Department, University of Pisa, Pisa, Italy

Correction notice This article has been corrected since it was published Online First. Labelling error in figure 2 was corrected and text within table 2 was updated. Due to the updated text in table 2 some minor corrections has been made throughout the manuscript.

Twitter Follow Martijn A Spruit @pulmonary_rehab and Nicholas Hopkinson @COPDdoc

Acknowledgements The authors thank Professor R Aarts, Dr X Long, Dr J W Bikker, Professor R L ZuWallack for the useful input provided to the manuscript.

Contributors GS, MAS, ACdB, conceived and designed the analysis. GS analysed the data and drafted the first version of the manuscript. MAS and $A C \mathrm{CB}$. provided advice at all stages of the analysis. ACdB provided statistical support. MAS, JA, RPB, PMAC, CFC, RWC, DD-G, SD, JG-A, AJRvG, MG, NAH, KH, NSH, DJ, MK, AK, JDL, HM, FM WD-CM, ZJMCK, RM, DM, FP, SJS, FWJMS, RT-S, BV, BW, HW, EFMW and SZ. contributed to the acquisition of data in each centre included and/or participated in the critical revision of the manuscript. All authors accepted the final version of the manuscript.

Funding The Eindhoven-based authors and the CIRO-based authors gratefully acknowledge the financial support received by the iCare4COPD Project of Agentschap NL under Contract PNE101005. The Basel-based authors gratefully acknowledge the financial support received by the foundations 'Gottfried und Julia Bangerter-Rhyner-Stiftung', 'Freiwillige Akademische Gesellschaft Basel' and 'Forschungsfonds der Universität Basel'.

Competing interests None declared.

Patient consent Obtained.

Ethics approval The data collection was conducted in accordance with the declaration of Helsinki and approved by ethics committees at each of the participating centres, according to local regulations.

Provenance and peer review Not commissioned; externally peer reviewed.

\section{REFERENCES}

1 Watz H, Pitta F, Rochester CL, et al. An official European Respiratory Society statement on physical activity in COPD. Eur Respir J 2014;44:1521-37.

2 Gimeno-Santos E, Frei A, Steurer-Stey C, et al. Determinants and outcomes of physical activity in patients with COPD: a systematic review. Thorax 2014;69:731-9.

3 Agusti A, Hedner J, Marin J, et al. Night-time symptoms: a forgotten dimension of COPD. Eur Respir Rev 2011;20:183-94.

4 Kinsman RA, Yaroush R, Fernandez E, et al. Symptoms and experiences in chronic bronchitis and emphysema. Chest. 1983;83:755-61.

5 Sadeh $A$. The role and validity of actigraphy in sleep medicine: an update. Sleep Med Rev 2011;15:259-67.

6 Kelly JM, Strecker RE, Bianchi MT. Recent developments in home sleep-monitoring devices. ISRN Neurol 2012;2012:1-10.

7 Martin JL, Hakim AD. Wrist actigraphy. Chest J 2011;139:1514-27.
8 Nunes DM, de Bruin VM, Louzada FM, et al. Actigraphic assessment of sleep in chronic obstructive pulmonary disease. Sleep Breath 2013;17:125-32.

9 Hartman JE, Prinzen J, van Lummel RC, et al. Frequent sputum production is associated with disturbed night's rest and impaired sleep quality in patients with COPD. Sleep Breath 2015;19:1125-33.

10 McNicholas WT, Verbraecken J, Marin JM. Sleep disorders in COPD: the forgotten dimension. Eur Respir Rev 2013;22:365-75.

11 Pauwels RA, Buist AS, Calverley PM, et al. Global strategy for the diagnosis, management, and prevention of chronic obstructive pulmonary disease. Am J Respir Crit Care Med 2001;163:1256-76.

12 Bestall J, Paul EA, Garrod R, et al. Usefulness of the Medical Research Council (MRC) dyspnoea scale as a measure of disability in patients with chronic obstructive pulmonary disease. Thorax 1999:54:581-6.

13 BaHammam A, Alrajeh M, Albabtain M, et al. Circadian pattern of sleep, energy expenditure, and body temperature of young healthy men during the intermittent fasting of Ramadan. Appetite 2010;54:426-9.

14 Al Otair H, Al-shamiri M, Bahobail M, et al. Assessment of sleep patterns, energy expenditure and circadian rhythms of skin temperature in patients with acute coronary syndrome. Med Sci Monit 2011:17:CR397.

15 Yilmaz D, Dodd J, Shrikrishna D, et al. Changes in physical activity and sleep duration following hospitalisation with acute exacerbation of COPD. Eur Respir 2012;40(Suppl 56):P614.

16 Egan $C$, Deering BM, Blake $C$, et al. Short term and long term effects of pulmonary rehabilitation on physical activity in COPD. Respir Med 2012;106:1671-9.

17 Di Marco F, Terraneo S, Roggi MA, et al. Physical activity impairment in depressed COPD patients. Respir Care 2014;59:726-34

18 Teller A. A platform for wearable physiological computing. Interact Comput 2004;16:917-37. http://dx.doi.org/10.1016/j.intcom.2004.08.004

19 Troosters T, Sciurba F, Battaglia S, et al. Physical inactivity in patients with COPD, a controlled multi-center pilot-study. Respir Med 2010;104:1005-11.

20 Sharif MM, BaHammam AS. Sleep estimation using BodyMedia's SenseWear armband in patients with obstructive sleep apnea. Ann Thorac Med 2013;8:53.

21 Watz H, Waschki B, Meyer T, et al. Physical activity in patients with COPD. Eu Respir J 2009;33:262-72.

22 Garber CE, Blissmer B, Deschenes MR, et al. American College of Sports Medicine position stand. Quantity and quality of exercise for developing and maintaining cardiorespiratory, musculoskeletal, and neuromotor fitness in apparently healthy adults: guidance for prescribing exercise. Med Sci Sports Exercise 2011;43:1334-59.

23 Baron KG, Reid KJ, Zee PC. Exercise to improve sleep in insomnia: exploration of the bidirectional effects. J Clin Sleep Med 2013;9:819-24.

24 Lambiase MJ, Gabriel KP, Kuller LH, et al. Temporal relationships between physical activity and sleep in older women. Med Sci Sports Exerc 2013;45

25 Dzierzewski JM, Buman MP, Giacobbi PR, et al. Exercise and sleep in communitydwelling older adults: evidence for a reciprocal relationship. J Sleep Res 2014;23:61-8.

26 Goodnight JH. Tests of hypotheses in fixed effects linear models. Commun Stat Theory Methods 1980;9:167-80.

27 Nelson ME, Rejeski WJ, Blair SN, et al. Physical activity and public health in older adults: recommendation from the American College of Sports Medicine and the American Heart Association. Circulation 2007;116:1094.

28 George CF, Bayliff CD. Management of insomnia in patients with chronic obstructive pulmonary disease Drugs 2003;63:379-87.

29 Dodge R, Cline MG, Quan SF. The natural history of insomnia and its relationship to respiratory symptoms. Arch Intern Med 1995;155:1797-800.

30 Weitzenblum E, Chaouat A. Sleep and chronic obstructive pulmonary disease. Sleep Med Rev 2004:8:281-94.

31 Klink ME, Dodge R, Quan SF. The relation of sleep complaints to respiratory symptoms in a general population. Chest 1994;105:151-4.

32 Collop NA, Adkins D, Phillips BA. Gender differences in sleep and sleep-disordered breathing. Clin Chest Med 2004;25:257-68.

33 Vgontzas AN, Bixler EO, Kales A, et al. Validity and clinical utility of sleep laboratory criteria for insomnia. Inter J Neurosci 1994;77:11-21.

34 Scharf SM, Maimon N, Simon-Tuval T, et al. Sleep quality predicts quality of life in chronic obstructive pulmonary disease. Int J Chron Obstruct Pulmon Dis 2011;6:1-12.

35 Nunes DM, Mota RMS, de Pontes Neto OL, et al. Impaired sleep reduces quality of life in chronic obstructive pulmonary disease. Lung 2009;187:159-63.

36 Goldman SE, Stone KL, Ancoli-Israel S, et al. Poor sleep is associated with poorer physical performance and greater functional limitations in older women. Sleep 2007;30:1317-24.

37 Dam TTL, Ewing S, Ancoli-Israel S, et al. Association between sleep and physical function in older men: the osteoporotic fractures in men sleep study. J Am Geriatr Soc 2008:56:1665-73.

38 Wesensten NJ, Balkin TJ, Belenky G. Does sleep fragmentation impact recuperation? A review and reanalysis. J Sleep Res 1999:8:237-45.

39 Demeyer $\mathrm{H}$, Burtin C, Van Remoortel H, et al. Standardizing the analysis of physical activity in patients with COPD following a pulmonary rehabilitation program. Chest 2014:146:318-27.

40 Soler $\mathrm{X}$, Gaio $\mathrm{E}$, Powell FL, et al. High prevalence of obstructive sleep apnea in patients with moderate to severe chronic obstructive pulmonary disease. Ann Am Thorac Soc 2015;12:1219-25. 